\title{
CALIBRATION AND IMAGE RECONSTRUCTION FOR THE HURRICANE IMAGING RADIOMETER (HIRAD)
}

\author{
Christopher Ruf $*^{1}$, J. Brent Roberts ${ }^{2}$, Sayak Biswas ${ }^{3}$, Mark W. James ${ }^{2}$, and Timothy Miller ${ }^{2}$ \\ ${ }^{1}$ AOSS Dept., University of Michigan, Ann Arbor, MI 48109 USA \\ ${ }^{2}$ NASA, Marshall Space Flight Center, Huntsville, AL 35805 USA \\ ${ }^{3}$ CFRSL, University of Central Florida, Orlando. FL 32816 USA \\ *cruf@umich.edu, +1-734-764-6561
}

\section{INTRODUCTION}

The Hurricane Imaging Radiometer (HIRAD) is a new airborne passive microwave synthetic aperture radiometer designed to provide wide swath images of ocean surface wind speed under heavy precipitation and, in particular, in tropical cyclones. It operates at 4, 5, 6 and $6.6 \mathrm{GHz}$ and uses interferometric signal processing to synthesize a pushbroom imager in software from a low profile planar antenna with no mechanical scanning. HIRAD participated in NASA's Genesis and Rapid Intensification Processes (GRIP) mission during Fall 2010 as its first science field campaign. HIRAD produced images of upwelling brightness temperature over a $\sim 70 \mathrm{~km}$ swath width with $\sim 3 \mathrm{~km}$ spatial resolution. From this, ocean surface wind speed and column averaged atmospheric liquid water content can be retrieved across the swath. The calibration and image reconstruction algorithms that were used to verify HIRAD functional performance during and immediately after GRIP were only preliminary and used a number of simplifying assumptions and approximations about the instrument design and performance. The development and performance of a more detailed and complete set of algorithms are reported here.

\section{HIRAD INSTRUMENT OVERVIEW}

The HIRAD planar antenna is composed of linear arrays of stacked multi-resonant radiators, operating at 4, 5, 6, and $6.6 \mathrm{GHz}$ [1]. Each linear array, viewed as an individual fan beam antenna element, is placed in an optimum thinned array configuration to produce the interferometer baselines needed for aperture synthesis. Individual receivers after each fan beam element contain internal hot and cold calibration loads. A single correlated noise diode is also distributed to all receivers to calibrate the interferometric correlation measurements [2]. HIRAD's major components consist of the integrated stacked patch antenna array, analog receivers with integrated calibration sources, real time digital signal processing for frequency sub-banding, RFI mitigation and interferometric cross-correlation, command and data handling (C\&DH), Local Oscillator (LO) and noise diode 
(for calibration) distribution, and the Power Distribution Unit (PDU), which includes thermal control for the instrument.

\section{CALIBRATION ALGORITHM}

The basic signal flow through the radiometer for self-correlation measurements made by individual HIRAD receivers is shown in Fig. 1.

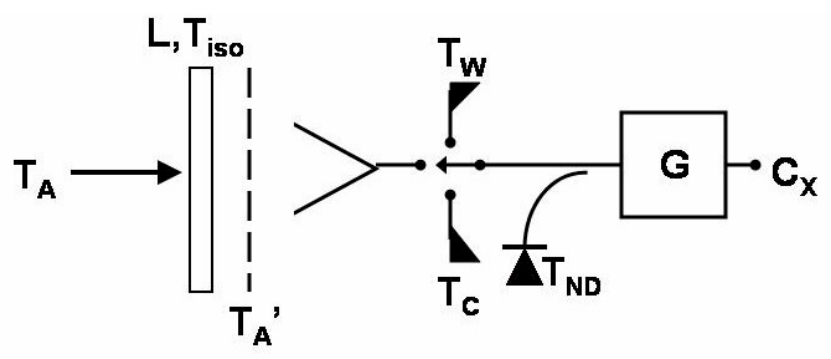

Figure 1. Signal flow diagram for HIRAD self correlation measurements.

In Fig. 1, $\mathrm{T}_{\mathrm{A}}$ is the incident brightness temperature weighted by the antenna pattern of an individual HIRAD antenna, $\mathrm{L}$ is the net transmissivity of the lossy radome and antenna, $\mathrm{T}_{\text {iso }}$ is the physical temperature of the radome and antenna, $\mathrm{T}_{\mathrm{A}}$ ' is the effective antenna temperature after propagation through the radome and antenna (including self emission by the lossy elements), $\mathrm{T}_{\mathrm{W}}$ and $\mathrm{T}_{\mathrm{C}}$ are the brightness temperatures of the internal warm and cold calibration loads, $\mathrm{T}_{\mathrm{ND}}$ is the increase in brightness temperature due to the noise diode, $\mathrm{G}$ is the receiver gain in units of Kelvins/counts, and $C_{X}$ is the raw $2^{\text {nd }}$ moment counts when the system is in state $X$. Possible system states include: $\mathrm{X}=\mathrm{A}$ (viewing the antenna), W (warm load), C (cold load), W+ND (warm load with noise diode on), and C+ND (cold load with noise diode on).

The $\mathrm{T}_{\mathrm{A}}$ calibration algorithm is given be

where

$$
\mathrm{T}_{\mathrm{A}}=\left[\mathrm{T}_{\mathrm{A}}-(1-\mathrm{L}) \mathrm{T}_{\mathrm{iso}}\right] \mathrm{L}^{-1}
$$

and

$$
\mathrm{G}=\left(\mathrm{T}_{\mathrm{W}}-\mathrm{T}_{\mathrm{C}}\right) /\left(\mathrm{C}_{\mathrm{W}}-\mathrm{C}_{\mathrm{C}}\right)
$$

$$
\mathrm{T}_{\mathrm{A}}{ }^{\prime}=\mathrm{T}_{\mathrm{W}}-\mathrm{dT}_{\mathrm{RX}}-\left(\mathrm{C}_{\mathrm{W}}-\mathrm{C}_{\mathrm{A}}\right) \mathrm{G}
$$

Equations (1)-(3) constitute the zero’th Visibility (V0) calibration algorithm. V0 corresponds to the average brightness temperature over the complete cross-track swath of the imager. 
The basic signal flow through the radiometer for cross-correlation measurements made by pairs of HIRAD receivers is shown in Figure 2.

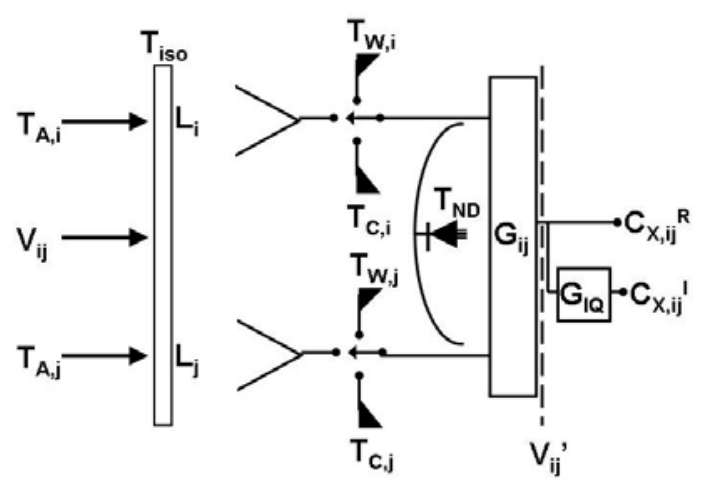

Figure 2. Signal flow diagram for HIRAD cross correlation measurements.

In Fig. 2, incident $\mathrm{T}_{\mathrm{A}}$ signals enter the $\mathrm{i}^{\text {th }}$ and $\mathrm{j}^{\text {th }}$ channels of HIRAD. The antenna transmissivity, $\mathrm{L}$, and the warm and cold reference brightness temperatures, $\mathrm{T}_{\mathrm{W}}$ and $\mathrm{T}_{\mathrm{C}}$, are in general different for each channel. A single noise diode is common to all ten HIRAD channels to provide a correlated signal, but its additive brightness temperature is in general different for each channel. $G_{i j}$ is the cross-correlation gain for the $i^{\text {th }}$ and $j^{\text {th }}$ channels. $C_{X, i j}{ }^{R}$ and $C_{X, i j}{ }^{I}$ represent the real and imaginary components of the raw cross-correlation counts when the system is in state $\mathrm{X}$, and $\mathrm{G}_{\mathrm{IQ}}$ represents the gain imbalance between the two complex components.

The Visibility calibration algorithm is given by

and

$$
V_{i j}{ }^{R}=\left(C_{A, i j}{ }^{R}-C_{0, i j}{ }^{R}\right) G_{i j} L_{i j}{ }^{-1}
$$

$$
\mathrm{V}_{\mathrm{ij}}^{\mathrm{I}}{ }^{\mathrm{I}}=\left(\mathrm{C}_{\mathrm{A}, \mathrm{ij}}^{\mathrm{I}}-\mathrm{C}_{0, \mathrm{ij}}{ }^{\mathrm{I}}\right) \mathrm{G}_{\mathrm{IQ}} \mathrm{G}_{\mathrm{ij}} \mathrm{L}_{\mathrm{ij}}{ }^{-1}
$$

where the cross-correlation gain is found as the geometric mean of the appropriate pair of self-correlation gains. If $\mathrm{Gi}$ is the self-correlation gain for receiver $\mathrm{i}$, given by (5), then

$$
G_{i j}=\left(G_{i} G_{j}\right)^{1 / 2}
$$

Equations (4)-(5) constitute the non-zero Visibility calibration algorithm.

\section{EXAMPLES OF CALIBRATION PERFORMANCE}

Time series plots of the calibrated zero'th visibility, V0, measurements made by HIRAD at $5 \mathrm{GHz}$ are shown in Fig. 3 for two airborne flights. The total passband is divided digitally into 16 subbands to aid in the detection and 
mitigation of radio frequency interference, and the individual V0 values for each subband are shown. The NEDT noise level is high for the individual subband data and is reduced when they are averaged together. The left plot shows V0 during a test flight made on 11 March 2010 during engineering commissioning. The brightness at the beginning of the flight is high while the aircraft is over land. V0 drops rapidly at the coastal crossing, then remains low and steady during the flight segment over calm, open water in the Gulf of Mexico. The final portion of the left hand plot shows the rise in V0 as the plan begins to cross back over land. The right hand plot shows V0 during the Hurricane Karl overpass on 16 September 2010. Very high V0 levels at the beginning and end correspond to over-land flight segments. Rapidly varying V0 values in the middle correspond to overpasses of Karl, together with aircraft bank maneuvers between overpass flight legs. Example images of the resolved cross track variations in brightness temperature across the swath and vs. frequency will also be presented at IGARSS.
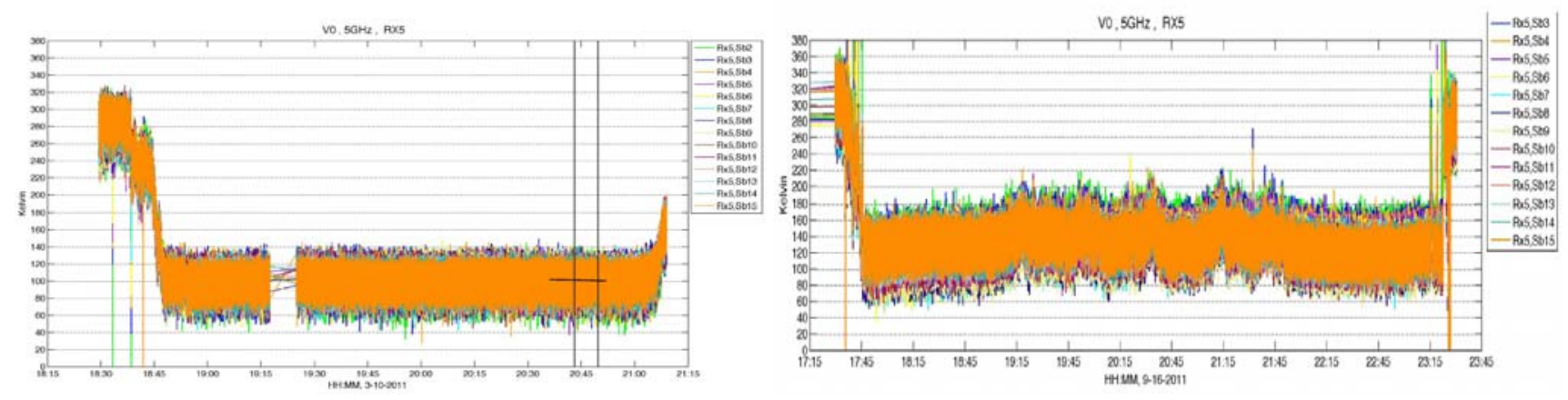

Figure 3. Time series plots of the average brightness temperature across the HIRAD swath at $5 \mathrm{GHz}$ for two aircraft flights. (left) 11 March 2010 test flight over clear, calm ocean. (right) GRIP overpass of Hurricane Karl on 16 September 2010.

\section{REFERENCES}

[1] Bailey, M.C., R. Amarin, J. Johnson, P. Nelson, M. James, D. Simmons, C. Ruf, L. Jones and X. Gong, “Multi-Frequency Synthetic Thinned Array Antenna for the Hurricane Imaging Radiometer," Trans. Antennas Propagat., 58(8), doi:10.1109/TAP.2010.2050453, 2010.

[2] Ruf, C., R. Amarin, M.C. Bailey, B. Lim, R. Hood, M. James, J. Johnson, L. Jones, V. Rohwedder and K. Stephens, "The hurricane imaging radiometer - An octave bandwidth synthetic thinned array radiometer," Proc. IGARSS 2007, Barcelona, 23-27 July 2007. 